\title{
A new polymorphism on chromosome 6 associated with bolting tendency in sugar beet
}

\author{
Chiara Broccanello ${ }^{1}$, Piergiorgio Stevanato ${ }^{1 *}$, Filippo Biscarini ${ }^{2}$, Dario Cantu ${ }^{3}$ and Massimo Saccomani ${ }^{1}$
}

\begin{abstract}
Background: Premature flowering or bolting is an undesirable characteristic that causes severe sugar yield losses and interferes with harvesting. Vernalization is a prerequisite for the floral induction, achieved by exposure to low temperatures for 10-14 weeks. This process is also controlled by other environmental factors, such as long daylight photoperiods and a combination of genetic factors. The objective of this study was the identification of new genetic polymorphisms linked to bolting tendency in sugar beet.

Results: Two pollinators characterized by low and high bolting tendency were subjected to RAD-sequencing in order to detect discriminating SNPs between lines. 6,324 putative SNPs were identified. Of these, 192 were genotyped in a set of 19 pollinators, each comprising bolted and non-bolted individuals, for a total of 987 samples. Among the 192 candidate SNPs, the strongest overall association was found for SNP183 on chromosome 6 ( $p$-value = $1.246 \quad 10^{-13}$ ). The association between SNP183 and bolting tendency was then confirmed in an independent population of 730 plants from 11 breeding lines ( $p$-value $=0.0061)$. SNP183 is located in the intron of Bv_22330_orky, a sugar beet homolog of a matrix metalloproteinase (MMP) gene that could be implied in flowering in Arabidopsis thaliana.

Conclusion: Our data support a significant association between an intronic SNP in the MMP gene located on chromosome 6 and the regulation of bolting tendency in sugar beet. The newly identified locus supports the polygenic nature of flowering control. The associated marker can be used to design SNP panels for the discrimination of bolters and non-bolters, to be used in sugar beet breeding programs for the development of improved germplasm with low bolting tendency.
\end{abstract}

Keywords: Bolting tendency, RAD sequencing, SNP association, Molecular breeding, Beta vulgaris

\section{Background}

For an effective genetic improvement of sugar beet (Beta vulgaris L.) it is critical to gain a better understanding of the biological processes behind the switch from vegetative growth to floral induction [1]. Premature flowering or bolting is an undesirable characteristic that causes severe sugar yield losses and interferes with harvesting [2]. Under field conditions, cultivated sugar beet is a biennial plant that requires two full growing seasons to switch from the vegetative phase to bolting. Vernalization is a prerequisite for the floral induction, achieved by exposure to low

\footnotetext{
* Correspondence: stevanato@unipd.it

'DAFNAE, Dipartimento di Agronomia Animali Alimenti Risorse Naturali e Ambiente, Università degli Studi di Padova, viale dell'Università 16, 35020 Legnaro, PD, Italy

Full list of author information is available at the end of the article
}

temperatures for 10-14 weeks [3]. This process is also controlled by other environmental factors, such as long daylight photoperiods and a combination of genetic factors [4]. Sugar beet bolting tendency is known to be influenced genetically by the $B$ locus, mapped on chromosome 2 [5-7]. Homozygous plants at the $B$ locus $(B B)$ initiate bolting under long day conditions whereas plants carrying recessive alleles in the homozygous state $(b b)$ need vernalization for floral induction. Environmental and genetic factors strongly influence heterozygous plants $(B b)$ that show a more complex behaviour $[5,6,8,9] . B b$ plants bolting without vernalization show a delay in bolting time compared to $B B$ individuals [10]. The $B$ locus was recently found to correspond to the BOLTING TIME CONTROL 1 (BTC1) gene. Biennial plants, which do not flower without a period of vernalization, carry a partial 
loss of function BTC1 allele. A second locus (B2) mapped on chromosome 9 and acting epistatically with the $B$ locus was also associated with bolting behaviour. $B v B B X 19$, encoding a DOUBLE B-BOX TYPE ZINC FINGER protein B-box transcription factor was found to underlie the $B 2$ locus $[11,12]$.

Given the known complexity of floral regulation in model species it is likely that additional genes influence bolting behavior in sugar beet [2]. In Arabidopsis thaliana, FLOWERING LOCUS C (FLC), CONSTANS (CO), and FLOWERING LOCUS T (FT) are key genes controlling flowering. Similar genes also exist in sugar beet: $B v F L 1$ on chromosome 6 [13], BvCOL1 on chromosome 2 [14], and $B v F T 1$ and $B v F T 2$ on chromosomes 9 and 4, respectively [15]. BvFT1 and BvFT2 are major regulators of bolting in beet [15] and act downstream of the $B$ and $B 2$ locus genes $B T C 1$ and $B v B B X 19[12,16]$. The $F L C$-like gene $B v F L 1$ is a floral repressor. Its expression is down regulated during a prolonged cold period under long daylight condition [13]. Similarly, CO-like gene $B v C O L 1$ reinforces the late flowering phenotype [14]. The functional role of the $F L C$ like and $\mathrm{CO}$-like genes suggests a partial evolutionary conservation in the regulation of floral transition between Arabidopsis and sugar beet [17].

Due to the highly complex interactions between genotype and environment, initial progress in bolting resistance was obtained by selecting varieties specific for the climates where they would be grown [18]. Selection was based solely on phenotypic observations by discarding early bolting plants, which were considered dominant heterozygous or homozygous at the $B$ locus.

The use of molecular markers can facilitate the detection of unfavorable alleles linked to the bolting tendency, allowing for earlier and more precise selection of nonbolters. Single Nucleotide Polymorphisms (SNPs) are ideal markers for this kind of work since they are spread throughout the genome and represent $90 \%$ of sequence variation among plants [19]. SNP markers have already been applied in sugar beet breeding programs [20]. Additionally, technical progress and the cost reduction of next-generation sequencing (NGS) technology can facilitate the identification of a large number of SNPs in any genomic region of interest [21, 22]. Among NGS techniques, Restriction-site Associated DNA (RAD) sequencing allows the discovery of several thousands of genetic variants adjacent to restriction enzyme cleavage sites across a target genome [5].

In this paper we suggest the identification of a new putative locus involved in the genetic determination of bolting tendency in sugar beets. Two sugar beet pollinators, P1 and P2, characterized respectively by early- and latebolting habit were subjected to RAD-SNP discovery. 192 SNPs were selected for further SNP association analysis. These SNPs were genotyped on a set of 19 pollinators, each comprising bolted and non-bolted individuals, for a total of 987 samples. The association between SNP genotypes and bolting tendency was tested by fitting one SNP at a time in a logistic regression model. A SNP marker associated with bolting tendency was located on chromosome 6. This SNP was then tested in an independent sugar beet population. The novel associated polymorphism provides further indication of the polygenic nature of bolting tendency in sugar beet.

\section{Results \\ SNP discovery}

RAD sequencing of the two DNA bulks, including (respectively) 4 non-bolted P1 and four bolted P2 plants, produced 96,822,109 raw reads of which $81,031,436(84 \%)$ were of high quality (longer than $100 \mathrm{nt}$ ) with an average length of $103.26 \mathrm{nt}$. RAD paired end sequence assembly was created using the P1 reads. Sequences from the P2 bulk were aligned to reference assembly for P1 using Bowtie (parameter: bowtie -f $-\mathrm{v} 1$ ). The aligned reads revealed a total of $288,843(\sim 150 \times$ coverage) unique consensus RAD tags common between the two bulks. The SNP discovery pipeline highlighted a total of 6,324 SNPs. Contigs were aligned to the sugar beet reference genome (RefBeet-1.1; http:// bvseq.molgen.mpg.de) to exclude SNPs with nearby flanking polymorphisms within $50 \mathrm{bp}$. A total of 192 polymorphic SNP between bulks, randomly distributed within and across all chromosomes, were selected for the SNP association analysis. The array of 192 SNPs used in this study along with their corresponding sequences are available as Additional file 1: Table S1.

\section{SNP genotyping and association mapping}

192 SNPs were genotyped on 987 samples from 19 pollinators each comprising both non-bolted and bolted individual plants. The relationship between SNP genotypes and bolting phenotypes was modeled with logistic regression. Among the 192 candidate SNPs, the only significant association was found for SNP183 on chromosome $6\left(P=1.2 \cdot 10^{-13}\right)$. Table 1 reports the analysis of deviance from the logistic regression model (see equation 1 in Methods section) for SNP183. From logistic regression, the probabilities for each plant, based on the population they belong to and their genotype at SNP183, of either showing or not bolting tendency were obtained. Figure 1 shows the distribution of such probabilities for the three genotypes at locus 183 .

To obtain the NCBI Reference Sequence ID for SNP183, a 440 bp long segment centering on SNP183 was PCR amplified, sequenced by a Sanger sequencing platform (ABI 3730xl) and blasted on NCBI. The resulted NCBI ID was XM_010697593.1. 
Table 1 Analysis of deviance table for a logistic regression model with the effects of pollinator population (19 classes) and genotypes at SNP183 on chromosome 6

\begin{tabular}{|c|c|c|c|c|c|}
\hline & Df & Deviance & Residual Df & Residual Deviance & $p$-value \\
\hline NULL & & & 929 & 1286 & \\
\hline Population & 18 & 173.01 & 911 & 1113 & $2.3 \quad 10^{-27}$ \\
\hline SNP183 & 2 & 59.43 & 909 & 1053 & $1.210^{-13}$ \\
\hline
\end{tabular}

SNP183 was mapped in the sequence of the single intron present in the Bv_22330_orky gene and it was not mapped in any gene known to be involved in bolting (Christian Jung, pers. comm.). As shown in Methods, SNP183 does not cosegregate with the BTC1 locus on chromosome 2. In addition, though both on chromosome 6, SNP183 and BvFL1 are on different (not anchored) scaffolds (Bvchr6_un.sca007 and Bvchr6.sca027, respectively). Further studies are needed to clarify if SNP183 and BvFL1 could co-segregate.

The frequency of the CC genotype was significantly increased in the bolting group ( $17 \%$ vs. $5 \% ; P=4.4 \cdot 10^{-7}$ ), while the TT genotype was significantly higher in the non-bolting group (67 \% vs. $\left.49 \% ; P=1.8 \cdot 10^{-6}\right)$ (Table 2). The two alleles of the SNP183 and the flanking sequences on each side of the SNP are reported in Additional file 1: Table S1. The sequences of the primers and TaqMan probes designed for the detection of the SNP183 are also given in Additional file 2: Table S2.
The location of SNP183 along the Bv_22330_orky gene sequence is shown in Fig. 2. The total length covered by the coding exons is $133 \mathrm{bp}$ and $585 \mathrm{bp}$ and the total length of the intron is $419 \mathrm{bp}$.

Bv_22330_orky encodes a putative Matrix Metalloproteinase (MMP) causing late flowering and early senescence in Arabidopsis thaliana. In sugar beet, four genes are annotated as MMPs gelatinase A based on the recently annotated genome [23]: Bv5_099660_fneg, Bv1u_021120_ $y k m a, B v \_22320 \_w u o m$ and Bv_22330_orky.

Five MMPs similar to Bv_22330_orky were found in Arabidopsis thaliana by BLASTP homology searches, as already reported in Golldack et al. [24]). We constructed a phylogenetic tree based on the NJ (neighbour-joining) method, using the full-length protein alignment (Fig. 3). Phylogenetic analysis shows the tight clustering, in a separate clade, of Bv_22320_wuom and Bv_22330_orky with $100 \%$ bootstrap support.

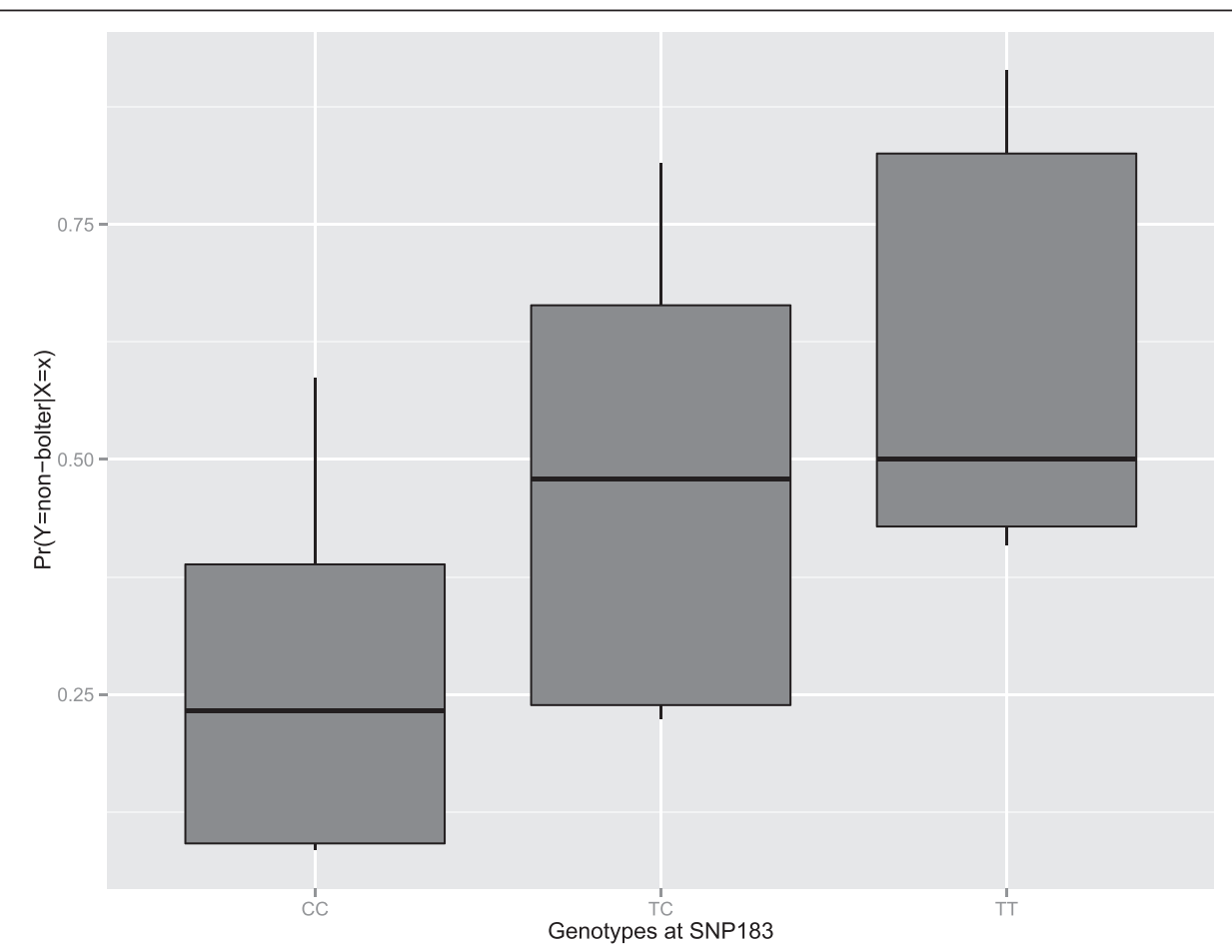

Fig. 1 Boxplot of the distribution of probabilities of showing either high or low bolting tendency for the three genotypes at SNP locus 183 (CC, CT, TT) based on a logistic regression model 
Table 2 Genotype frequencies of SNP183 on bolting and non bolting individuals

\begin{tabular}{|c|c|c|c|c|c|c|c|}
\hline & \multicolumn{2}{|l|}{ Bolting individuals $(n=436)$} & \multirow[t]{2}{*}{ Non bolting individuals $(n=495)$} & \multirow{2}{*}{$\begin{array}{l}x^{2} \\
n\end{array}$} & \multicolumn{3}{|c|}{$p$-value } \\
\hline & $n$ & $\%$ & & & $\%$ & & \\
\hline \multicolumn{8}{|c|}{ SNP183 } \\
\hline$\pi$ & 214 & 49 & & 332 & 67 & 22.8 & $1.810^{-6}$ \\
\hline TC & 150 & 34 & & 138 & 28 & 0.5 & 0.479 \\
\hline CC & 72 & 17 & & 25 & 5 & 25.5 & $4.4 \quad 10^{-7}$ \\
\hline
\end{tabular}

Testing SNP183 in an independent sugar beet population The SNP183 was genotyped in 730 individual plants from 11 breeding lines. A TaqMan assay was developed to discriminate rapidly and reliably between the $\mathrm{C}$ and $\mathrm{T}$ alleles at SNP183 locus. The frequency of the dominant $\mathrm{C}$ allele was $66 \%$ in the bolting group and $46 \%$ in the non-bolting group. Based on these results, individual plants carrying the $\mathrm{C}$ allele associated to high bolting tendency were discarded from the breeding program. The association between SNP genotype and bolting behavior was tested with a logistic regression model and was mildly significant $(P=0.0062)$.

\section{Discussion}

This study revealed a significant association between the polymorphism SNP183 on chromosome 6 and bolting tendency in sugar beet. The association was first detected in a population of 19 pollinators, where SNP discovery and association studies were carried out. Later, the association was tested in an independent population of 11 breeding lines. In both cases, the association between SNP183 genotypes and bolting behavior was significant. This suggests the presence of a new putative locus for bolting control on chromosome 6 of the sugar beet genome, which has not been reported, yet. This marker can be used in marker-assisted selection (MAS) programs to select for bolting resistance in sugar beets. MAS approaches to the reduction of bolting tendency are highly desirable in sugar beet breeding, since they are more efficient, faster, and often more reliable and less expensive than phenotypic selection, and allow to breed for complex traits like resistance to bolting. Bolting tendency is a complex trait controlled by

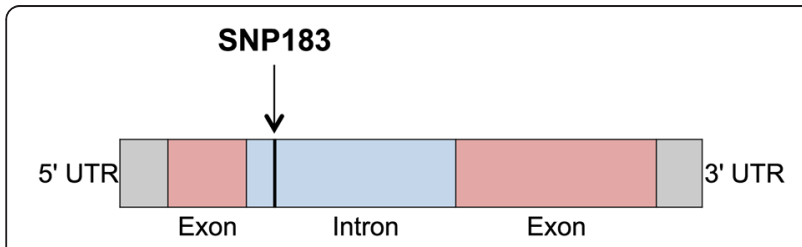

Fig. 2 Schematic representation of the Bv_22330_orky gene with the position of the SNP183 according to the reference genome (0096.scaffold00336: position 428612 to 430133; RefBeet-1.1; http://bvseq.molgen.mpg.de) environmental and developmental cues and multiple genetic loci [25]. The intricate network of regulatory pathways reflects complexity of the flowering process, with the vernalization, photoperiod, autonomous and gibberellic acid pathways and the circadian clock all contributing to the control of flowering [1, 26]. Given this complexity, multivariate statistical approaches to combine different sources of information are recommended for breeding applications to reduce bolting tendency in sugar beet. Previous attempts to model genomic predictions for binary traits in sugar beet have been reported [20, 27], and could be applied to the likewise binomially distributed bolting behavior. SNP183 can therefore potentially be used to design a SNP panel which includes polymorphisms from genomic associated with bolting tendency in sugar beet and that can differentiate bolters from non-bolters.

SNP183 was mapped to the intron sequence of the sugar beet gene $B v \_22330 \_o r k y$. While this gene may play a role in bolting control, which has not been previously reported in sugar beet, the SNP183 may actually be in linkage disequilibrium with neighbouring genes associated to bolting tendency. Besides being a marker linked to a gene involved in bolting behaviour, SNP183 -though less likely- could actually have a biological role itself: it can be a silent informative mutation that modifies splicing, if located in the donor/acceptor splice site; or it could affect the micro RNA binding.

Bv_22330_orky was found to code for a matrix metalloproteinase (MMP). MMPs are a family of zinc and calcium dependent proteases and are divided into three subfamilies: gelatinases, collagenases and stromelysins [28]. Human MMPs play important roles in many physiological

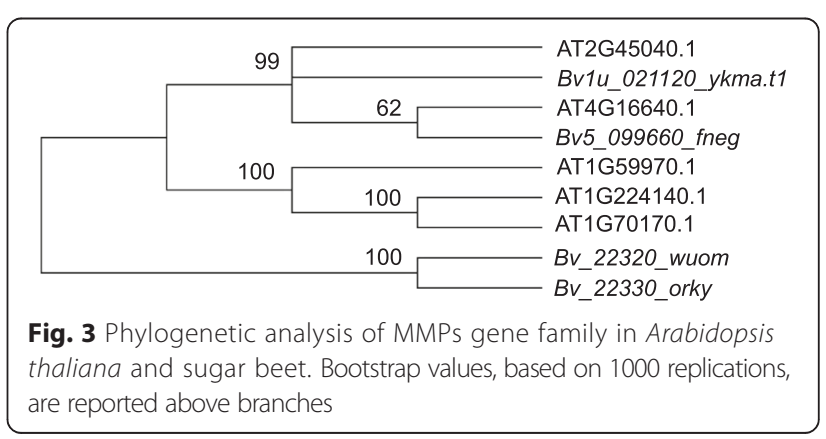


processes such as embryogenesis and organ morphogenesis. The unregulated MMPs activity is involved in the development of cancer, and neurodegenerative, cardiovascular and autoimmune disorders [29]. The diversity of functions inside mammalian MMPs derives from tandem duplication events and exon shuffling which took place during evolution. Most of the actual MMPs derive from a single gene cluster, conserved from amphibians to mammals. Plant MMPs are secreted during growth, development and stress response and play an important role in the degradation of extracellular matrix [30]. In Arabidopsis, MMPs is a family of proteins that could be implied in flowering [24] and, as it was found also in cucumber, are involved in the apoptosis [31]. In tobacco, they are expressed during senescence and the response to pathogens [32]. In sugar beet, we found two tandemduplicated MMP genes with $69 \%$ sequence similarity at DNA level. The gene duplication event, in Bv_22330_orky, led to the loss of the first $220 \mathrm{bp}$. This is also found in rice, where in duplicated blocks, DNA segment loss occurred with high frequency [33]. Tandem duplications are the most important events that generate new members of family proteins during evolution, generating novelty that may be selected in response to environmental changes [34].

Today, molecular markers are used to evaluate sugar beet germplasm only for the presence of annual bolters [3]. Several polymorphisms in BTC1 are able to discriminate between the annual or biennial habit of sugar beet [16]. However, these markers do not differentiate among biennial beets characterized by either high or low bolting tendency after exposure to a period of cold temperatures, suggesting that other (modifying) genes (and/or yet undiscovered polymorphisms in BTC1) affect bolting tendency in cultivated biennial sugar beets. Therefore, a next challenge is the discovery of additional DNA polymorphisms associated with this trait. As a first specimen of such polymorphism, SNP183 on chromosome 6 can be used -together with other- polymorphisms as a tool to improve selection efficiency and accelerate the development of novel sugar beet varieties displaying low-bolting tendency.

\section{Conclusions}

Our study provides indication for the association of a DNA polymorphism on chromosome 6 with bolting tendency in sugar beet. The results support the polygenic nature of flowering control in sugar beet confirming the importance of previously reported QTLs. The SNP183, together with other associated polymorphisms, could assist breeding programs aimed at developing germplasm with low bolting tendency. Further studies on this gene will provide new insights into genetic mechanisms of bolting, which are needed to breed for bolting resistance in sugar beet.

\section{Methods}

\section{Plant material}

The plant material used in this study was provided by the Department of Agronomy, Food, Natural Resources, Animals, and Environment, University of Padova (DAFNAE, Università degli Studi di Padova, Italy). For SNP discovery, two sugar beet pollinators, P1 and P2, characterized respectively by early- and late-bolting habit, were subjected to RAD-sequencing. The majority of P1 plants started to bolt 5 weeks from sowing while P2 plants started to bolt much later (at 15 weeks) after vernalization and in long-daylight conditions. Both P1 and P2 pollinators carrying the allele for biennial habit at the BTC1 locus in the homozygous state [16].

For SNP association analysis, 19 sugar beet pollinators segregating for bolting tendency were evaluated. Approximately 1000 seeds per pollinator were sown early (February 22, 2013) in a randomized block design at the Experimental Farm of the University of Padova. As expected, several plants for each pollinator died due to cold stress during the early seedling stage. The surviving plants were inspected every week for onset of bolting until June 30, 2013. Every week plants showing stem elongation were scored as bolting individuals while plants that did not show stem elongation were classified as non-bolting individuals [7]. A leaf sample was collected from each plant. Plants were divided into a group of non-bolted individuals and a group of bolted individuals for a total of 987 samples (Table 3).

\section{SNP discovery}

High-quality genomic DNA, from the parental lines (P1 and P2) used for discovery of markers, was extracted from leaf tissue following the procedure described by Stevanato et al. [35]. DNA samples were quantified on an Agilent 2200 TapeStation (Agilent Technologies, Santa Clara, USA). RAD sequencing was performed on two DNA bulks containing respectively 4 non-bolted P1 and 4 bolted P2 plants. All steps, including library preparation, were carried out by Floragenex (Eugene, OR) following the protocol described by Baird et al. [22] and Stevanato et al. [35]. Sequencing was performed on an Illumina HiSeq2000 platform. Raw sequences were trimmed to remove low quality reads, resulting from base-duplication calling, and those that lacked a correct barcode. The reads obtained were compared between the two bulks and the monomorphic sequences were removed. Only sequences with one nucleotide variation between the high and low bolting tendencies and mapped to the reference genome (version RefBeet-1.1; http://bvseq.molgen.mpg.de) were retained.

\section{SNP genotyping and association mapping}

A set of 192 randomly distributed SNPs was selected for SNP association analysis. These SNPs were tested on a 
Table 3 Sugar beet pollinators used for SNP association analysis

\begin{tabular}{|c|c|c|c|}
\hline Name & Total number of individuals ( $\mathrm{n}$ ) & Number of bolting individuals ( $\mathrm{n}$ ) & Number of non-bolting individuals (n) \\
\hline 101 & 20 & 10 & 10 \\
\hline 102 & 20 & 10 & 10 \\
\hline 103 & 20 & 10 & 10 \\
\hline 104 & 88 & 13 & 75 \\
\hline 105 & 90 & 15 & 75 \\
\hline 106 & 88 & 29 & 59 \\
\hline 107 & 47 & 10 & 37 \\
\hline 108 & 94 & 29 & 65 \\
\hline 109 & 20 & 10 & 10 \\
\hline 110 & 95 & 65 & 30 \\
\hline 111 & 20 & 10 & 10 \\
\hline 112 & 20 & 10 & 10 \\
\hline 113 & 94 & 64 & 30 \\
\hline 114 & 96 & 66 & 30 \\
\hline 115 & 20 & 10 & 10 \\
\hline 116 & 20 & 10 & 10 \\
\hline 117 & 20 & 10 & 10 \\
\hline 118 & 95 & 64 & 31 \\
\hline 119 & 20 & 10 & 10 \\
\hline Total & 987 & 455 & 532 \\
\hline
\end{tabular}

set of 19 pollinators, each comprising bolted and nonbolted individuals, for a total of 987 samples. Genotyping was performed using the Quant Studio $12 \mathrm{~K}$ Flex RealTime PCR System and Open Array technology (Life Technologies, CA, USA). The PCR reaction was prepared using $2.5 \mu \mathrm{l}$ of genomic DNA, at a concentration of $10 \mathrm{ng} \mu^{-1}$, added to $2.5 \mu \mathrm{l}$ of TaqMan OpenArray Genotyping Master Mix in a 384 well-plate. Samples from 384 well plate were loaded in the Open Array plate using the AccuFill system. The association between SNP genotypes and bolting tendency was tested by fitting one SNP at a time in a logistic regression model. A logit link function was used in a generalised linear model of the following form:

$$
\begin{aligned}
\operatorname{logit}\left(p\left(x_{i}\right)\right) & =\log \left(\frac{p\left(x_{i}\right)}{1-p\left(x_{i}\right)}\right) \\
& =\mu+\text { population }_{k}+z_{i j} S N P_{j}
\end{aligned}
$$

where $\operatorname{logit}\left(p\left(x_{i}\right)\right)$ is the log-odds of the probability $p$ for plant $i$ of having either high or low bolting tendency; $\mu$ is the overall trait mean, population $_{k}$ and $S N P_{j}$ are the fixed effects of plant population $k$ (19 classes) and SNP locus $j$, with $\mathrm{z}_{i j}$ an indicator variable for the genotype of plant $i$ at locus $j(0,1$ and 2 for $\mathrm{AA}, \mathrm{AB}$ and $\mathrm{BB})$.
Testing the detected association in an independent sugar beet population

The detected SNP-bolting association was tested in an independent annual beet population. The SNP183 was genotyped in 730 individual plants from 11 breeding lines. A TaqMan assay was developed to discriminate rapidly and reliably between the $\mathrm{C}$ and $\mathrm{T}$ alleles at SNP183 locus. All 730 plants were subjected to long photoperiod (16 h light / 8 h darkness) and $20.8 \%$ of the plants started to bolt from two weeks after sowing (bolting group), while $79.2 \%$ of plants did not show bolting behavior (non-bolting group). The association between SNP183 and bolting in the validation population was tested with the same logistic regression model used in the discovery population (see Equation (1)).

\section{Phylogenetic analysis}

Amino acid sequences were aligned with ClustalW [36] and phylogenetic tree was constructed using the neighbour-joining method as implemented in the software Mega version 6 [37, 38], with 1,000 bootstrap replicates.

\section{Availability of supporting data}

All supporting data are included as additional files. 


\section{Additional files}

Additional file 1: Table S1. Information on 192 SNPs used in the study. (XLSX $37 \mathrm{~kb}$ )

Additional file 2: Table S2. Sequences of the designed primers and TaqMan probes for detection of the SNP183. (DOC $28 \mathrm{~kb}$ )

\section{Abbreviations}

SNP: Single Nucleotide Polymorphism; RAD: Restriction-site Associated DNA

\section{Competing interests}

The authors declare that they have no competing interests.

\section{Authors' contributions}

CB and PS collected the data and wrote the manuscript. FB and DC helped with bioinformatics, data analysis and revision of the text. MS provided critical revision of the manuscript. All authors approved final version for publication.

\section{Acknowledgments}

The authors wish thanks to Dr. Andreas Müller (Strube Research GmbH \& Co. KG) and Dr. Enrico Biancardi (Former Director of CRA-CIN, Italy) for their critical reviews and remarks to improve the manuscript. The work was supported by ex-60 \% funds from the University of Padova, Italy.

\section{Author details}

'DAFNAE, Dipartimento di Agronomia Animali Alimenti Risorse Naturali e Ambiente, Università degli Studi di Padova, viale dell'Università 16, 35020 Legnaro, PD, Italy. ${ }^{2}$ FPTP, Fondazione Parco Tecnologico Padano, viale Einstein, Loc. Cascina Codazza, 26900 Lodi, Italy. ${ }^{3}$ Department of Viticulture and Enology, University of California, Davis, 2146 RMI North Building Davis, Davis, CA 95616, USA.

Received: 25 October 2015 Accepted: 26 November 2015

Published online: 07 December 2015

\section{References}

1. Pin PA, Zhang W, Vogt SH, Dally N, Büttner B, Schulze-Buxloh G, et al. The role of a pseudo-response regulator gene in life cycle adaptation and domestication of beet. Curr Biol. 2012;22:1095-101.

2. Jung C, Müller AE. Flowering time control and applications in plant breeding Trends Plant Sci. 2009;14:563-73.

3. Melzer S, Müller AE, Jung C. Genetics and genomics of flowering time regulation in sugar beet. In: Tuberosa R, Graner A, Frison E, editors. Genomics of plant genetic resources, vol. 2. Netherlands: Springer; 2014. p. 3-26.

4. Biancardi E, McGrath JM, Panella LW, Lewellen RT, Stevanato P. Sugar beet. In: Bradshow J, editor. Handbook of plant breeding, vol. 4. New York: Springer; 2010. p. 173-219.

5. Owen FV, Carsner E, Stout M. Photothermal induction of flowering in sugar beets. J Agr Res. 1940;61:101-24.

6. Abe J, Guan GP, Shimamoto Y. A gene complex for annual habit in sugar beet (Beta vulgaris L.). Euphytica. 1997:94:129-35.

7. Abe J, Guan GP, Shimamoto Y. A marker assisted analysis of bolting tendency in sugar beet (Beta vulgaris L.). Euphytica. 1997:94:137-44.

8. El-Mezawy A, Dreyer F, Jacobs G, Jung C. High-resolution mapping of the bolting gene B of sugar beet. Theor Appl Genet. 2002:105:100-5.

9. Shimamoto $Y$, Tanada T, Abe J. Analysis for bolting of sugar beet by means of the test crosses of biennial lines with annual line. Proc Japan Soc Sugar Beet Technol. 1990;32:134-7.

10. Boudry P, Wieber R, Saumitou-Laprade P, Pillen K, Van Dijk H, Jung C. Identification of RFLP markers closely linked to the bolting gene $B$ and their significance for the study of the annual habit in beets (Beta vulgaris L.). Theor Appl Genet. 1994;88:852-8.

11. Büttner B, Abou-Elwafa SF, Zhang W, Jung C, Müller AE. A survey of EMS-induced biennial Beta vulgaris mutants reveals a novel bolting locus which is unlinked to the bolting gene B. Theor Appl Genet. 2010;121:1117-31

12. Dally N, Xiao K, Holtgrawe D, Jung C. The B2 flowering time locus of beet encodes a zinc finger transcription factor. Proc Natl Acad Sci U S A. $2014 ; 111: 10365-70$
13. Reeves PA, He Y, Schmitz RJ, Amasino RM, Panella LW, Richards CM. Evolutionary conservation of the FLOWERING LOCUS C mediated vernalization response: evidence from the sugar beet (Beta vulgaris). Genetics. 2007;176:295-307.

14. Chia TY, Müller A, Jung C, Mutasa-Göttgens ES. Sugar beet contains a large CONSTANS-LIKE gene family including a CO homologue that is independent of the early bolting (B) gene locus. J Exp Bot. 2008;59:2735-48.

15. Pin PA, Benlloch R, Bonnet D, Wremerth-Weich E, Kraft T, Gielen JJL, et al. An antagonistic pair of FT homologs mediates the control of flowering time in sugar beet. Science. 2010;330:1397-400.

16. Mutasa-Göttgens ES, Qi A, Zhang W, Schulze-Buxloh G, Jennings A, Hohmann U, et al. Bolting and flowering control in sugar beet: relationships and effects of gibberellin, the bolting gene B and vernalization. AoB Plants. 2010;2010:plq012.

17. Frerichmann SL, Kirchhoff M, Müller AE, Scheidig AJ, Jung C, Kopisch-Obuch FJ. ECoTILLING in Beta vulgaris reveals polymorphisms in the FLC-like gene BVFL1 that are associated with annuality and winter hardiness. BMC Plant Biol. 2013;13:52.

18. Smit AL. Influence of external factors on growth and development of sugar beet (Beta vulgaris L.). Agricultural Res Reports 1983, Reports 914.

19. Sim SC, Durstewitz G, Plieske J, Wieseke R, Ganal MW, Van Deynze A, et al. Development of a large SNP genotyping array and generation of high-density genetic maps in tomato. PLoS One. 2012;7(7):e40563.

20. Biscarini F, Stevanato P, Broccanello C, Stella A, Saccomani M. Genomic predictions for binomial traits in sugar beet populations. BMC Genet. 2014;15:87.

21. Kumar S, Banks TW, Cloutier S. SNP discovery through next-generation sequencing and its applications. J Plant Genomics. 2012;2012:831460.

22. Baird NA, Etter PD, Atwood TS, Currey MC, Shiver AL, Lewis ZA, et al. Rapid SNP discovery and genetic mapping using sequenced RAD markers. PLoS One. 2008;3(10):e3376.

23. Dohm JC, Minoche AE, Holtgräwe D, Capella-Gutiérrez S, Zakrzewski F, Tafer $\mathrm{H}$, et al. The genome of the recently domesticated crop plant sugar beet (Beta vulgaris). Nature. 2013;505:546-9.

24. Golldack D, Popova OV, Dietz KJ. Mutation of the matrix metalloproteinase At2-MMP inhibits growth and causes late flowering and early senescence in Arabidopsis. J Biol Chem. 2002;277:5541-7

25. Engelmann K, Purugganan M. The molecular evolutionary ecology of plant development: Flowering Time in Arabidopsis thaliana. Adv Bot Res. 2006:44:507-26.

26. Mouradov A, Cremer F, Coupland G. Control of flowering time interacting pathways as a basis for diversity. Plant Cell. 2002;14(Suppl):S111-130.

27. Biscarini F, Marini S, Stevanato P, Broccanello C, Bellazzi R, Nazzicari N. Developing a parsimonious predictor for binary traits in sugar beet (Beta vulgaris). Mol Breed. 2015:35:10.

28. Borkakoti N. Structural studies of matrix metalloproteinases. J Mol Med. 2000:78:261-8

29. Murphy G, Nagase H. Progress in matrix metalloproteinase research. Mol Aspects Med. 2008;29:290-308.

30. Marino G, Funk C. Matrix metalloproteinases in plants: a brief overview Physiol Plant. 2012;145:196-202.

31. Delorme VG, McCabe PF, Kim DJ, Leaver CJ. A matrix metalloproteinase gene is expressed at the boundary of senescence and programmed cell death in cucumber. Plant Physiol. 2000;123:917-28.

32. Mandal MK, Fischer R, Schillberg S, Schiermeyer A. Biochemical properties of the matrix metalloproteinase NTMMP1 from Nicotiana tabacum cV. BY-2 suspension cells. Planta. 2010;232:899-910

33. Wang X, Shi X, Hao B, Ge S, Luo J. Duplication and DNA segmental loss in the rice genome: implications for diploidization. New Phytol. 2005;65:937-46.

34. Zhang J. Evolution by gene duplication: an update. Trends Ecol Evol. 2003;18:292-8

35. Stevanato P, Trebbi D, Panella L, Richardson K, Broccanello C, Pakish L, et al. Identification and validation of a SNP marker linked to the gene HsBvm-1 for nematode resistance in sugar beet. Plant Mol Biol Rep. 2015;33:474-9.

36. Thompson JD, Gibson T, Higgins DG. Multiple sequence alignment using ClustalW and ClustalX. Curr Protoc Bioinformatics. 2002;2:2.3.

37. Tamura K, Stecher G, Peterson D, Filipski A, Kumar S. MEGA6: molecula evolutionary genetics analysis version 6.0. Mol Biol Evol. 2013;30:2725-9.

38. Saitou N, Nei M. The neighbor-joining method: a new method for reconstructing phylogenetic trees. Mol Biol Evol. 1987;4:406-25. 